\title{
Le profil de l'apprenant numérique, du collège à l'université : le cas de Pau
}

Jean-François Ceci

Université de Pau et des Pays de l'Adour (France)

\author{
The digital learner profile, \\ from college to university: case of Pau
}

doi: $10.18162 / f p .2019 .485$
Une étude de terrain visant à cartographier les usages numériques formels et informels des apprenants, du collège à l'université, confirme que ces jeunes sont hyperconnectés. Cette hyperconnexion se manifeste

à travers un large éventail d'activités numériques génératrices d'apprentissages. Le présent article mettra l'accent sur la sensation d'apprendre avec le numérique, dans un contexte aussi bien formel qu'informel. Nous dresserons ensuite, s'il existe, un profil type de l'apprenant numérique, une personne aimant apprendre sur écrans et souhaitant voir se développer le numérique éducatif. Enfin, à l'aune de ces résultats, nous pourrons voir si l'École (au sens large de la $6^{e}$ à M2) s'est emparée du numérique pour faire apprendre. La partie empirique de cette étude est basée sur un pluralisme méthodologique, mêlant une approche qualitative et quantitative.

\section{Mots-clés}

Hyperconnexion, apprentissage formel, apprentissage informel, numérique éducatif, apprenant numérique.

\section{Abstract}

A sociological study with the aim to map the formal and informal digital practices of learners, from secondary school up to university, shows that young people are hyper-connected. This hyper-connectivity manifests itself through a large panel of digital activities, generating learning of different types. This paper focuses on the feeling of learning with digital, both in a formal and informal context. Then, if it exists, we will draw up a typical profile of the digital learner, a person who likes to learn on screens and wishing to see the development of digital education. Finally, by the yardstick of these results, we will be able to see if School (in the broad sense of the 6th to M2) has seized digital technology to teach. The

empirical part of this study is based on a methodological pluralism, combining a qualitative and quantitative approach.

\section{Keywords}

Hyperconnection, formal learning, informal learning, digital education, digital learner.

\section{Introduction}

En nous plaçant dansla perspective que nous propose Philippe Meirieu (1999), nous constatons que notre forme scolaire française n'a que peu évolué depuis l'abandon de « l'école mutuelle » telle que pratiquée par Johann Heinrich Pestalozzi (Buisson, 1911), au profit de « l'école normale » promulguée par le ministre François Guizot en 1833 : nous sommes passés d'une école de la formation de pairs à pairs (dite école mutuelle), de l'apprentissage collaboratif, de classes sans niveaux, à une forme scolaire de la transmission, normée : un enseignant, des élèves en classe d'âge, un enseignement identique pour tous. On parle à l'époque de "mécaniser l'éducation », alors que les résultats semblaient pourtant être au rendez-vous, tel que le constate le rapport de la commission de la Société des amis de l'éducation :

Nous avons remarqué tout d'abord [...] que les enfants de l'établissement de Pestalozzi apprennent, dans un temps très court, à épeler, à lire, à écrire et à calculer dans la plus grande perfection. Six mois suffisent pour les amener au degré où un instituteur de village aurait mis trois ans à les conduire [...] et que c'est à la méthode d'enseignement qu'il faut les attribuer. (Buisson, 1911)

L'innovation pédagogique, à la fois phénomène de mode et injonction sociétale ou institutionnelle, porte souvent sur une évolution de cette forme scolaire normée : d'une "pédagogie de la transmission » vers la mise en action de l'apprenant ${ }^{1}$ sur les connaissances à acquérir (pédagogie dite « nouvelle » ou « active »). 


\section{Cadre théorique}

Le numérique nous donne d'autres possibilités de faire société et l'école n'y échappe pas avec l'apparition du numérique éducatif tel que défini par Simon Collin et que nous adopterons dans son sens le plus large (Collin, 2013). Il est reconnu comme un puissant levier d'innovation pédagogique en brisant les frontières de la classe et du temps scolaire, en démultipliant les capacités créatives et co-créatives et permettant «de donner le goût d'apprendre » (Karsenti, 2016; Romero, Laferriere et Power, 2016). I1 crée ce que Michel Crozier et Erhard Friedberg (2014) appellent des "zones d'incertitudes ", c'està-dire des situations où une innovation produit de l'imprévisibilité, libérant un pouvoir d'initiative et d'action dans des systèmes jusqu'alors soumis à des modes de fonctionnement établis. Qu'est-il en train d'apparaitre sous nos yeux dans le système scolaire, quels sont les acteurs qui le portent et selon quelles logiques d'action? En effet, comme le mentionne Rebecca Eynon (2012) :

Le domaine du numérique en éducation n'est pas exempt de ce biais en cela que les chercheurs qui y œuvrent continuent d'être sommés de prouver le bénéfice, l'intérêt et la valeur ajoutée des technologies, à la fois pour légitimer des investissements financiers massifs ou pour justifier des attentes par rapport à une régénération de l'apprentissage scolaire par le biais du numérique. (Eynon, 2012, cité dans Collin, Guichon et Ntebutse, 2015)

L'état de la recherche montre que l'école doit évoluer d'une approche majoritairement centrée sur l'enseignement (transmission), à une approche ajoutant l'apprentissage par l'action et par les pairs (learning by doing). En effet, les nombreuses théories de l'apprentissage et auteurs autour du constructivisme, du socioconstructivisme et des pédagogies fonctionnant autrement que par instruction directe (Dewey, Freinet, Montessori, Pestalozzi, Vygotsky, Piaget...) abondent en ce sens. Il s'agit, entre autres, de faire une place importante aux activités collaboratives, à l'esprit critique, à « l'apprendre en faisant » tout en donnant davantage de sens aux enseignements et en les reliant au vécu des apprenants (Bandura et Locke, 2003). Nous pensons que cette évolution du système éducatif ne peut se faire sans une prise en compte des usages numériques des étudiants, notamment lors des apprentissages formels (en lien avec l'institution scolaire) ou informels (les autres) ${ }^{2}$. Le contexte socioculturel de l'apprenant doit également être étudié et relié aux pratiques numériques effectives puisque d'après le rapport de l'OCDE sur les "new millennium learners " (2008), le numérique est surtout utilisé hors contexte institutionnel. Qu'en est-il neuf ans après, l'école s'est-elle emparée du numérique? De même, d'après le rapport de l'UNESCO (Hamadache, 1993, p. 10), « c'est un fait que la plus grande partie des connaissances et des savoir-faire qu'acquiert un individu au cours de son existence se fait, dans un environnement non structuré, au moyen de ce mode d'éducation [informelle] ». Le contexte non institutionnel doit donc être analysé et comparé, en termes de pratiques numériques avec le contexte formel (institutionnel). Enfin, nos données nous permettront de dresser un début de profil social de l'apprenant avec le numérique, que nous nommerons par commodité l'apprenant numérique, une personne aimant apprendre sur écrans et souhaitant voir se développer le numérique éducatif : en quelque sorte la version 2017 du « new millenium learner » précité. 
Nous positionnons cet article à la croisée de la sociologie des usages et de l'éducation et des sciences de l'éducation. La sociologie des usages et de l'éducation est convoquée pour étudier ce que les gens font effectivement avec les objets techniques à l'école et en dehors, ainsi que la construction identitaire à l'ère du numérique. Les sciences de l'éducation nous permettent d'aborder l'apprendre avec le numérique. Nous effectuons aussi quelques incursions en sic et dans le domaine des neurosciences, sur les mêmes thématiques.

\section{Méthodologie}

Le cadre méthodologique se base sur une sociologie critique telle que décrite par Jean De Munck (2011, p. 2). Pour lui :

une sociologie est critique lorsquelle cherche consciemment et explicitement à se situer à la jonction des trois dimensions constitutives [...] la première tâche $[\ldots]$ est d'identifier le réel social [...] en mobilisant un appareil conceptuel adéquat, [...] La deuxième tâche est d'identifier des mal-fonctionnements ou des pathologies dans la société [...] [et la troisième de guider] une intervention dans la réalité sociale en vue de la transformer.

Une étude sociologique en cours (2016-2018) dans la région de Pau (France), visant à cartographier les usages numériques formels et informels des jeunes du collège à l'université, ainsi que les usages numériques de leurs enseignants, nous permet « d'identifier le réel social ». Nous étudierons ensuite les usages (la deuxième tâche) afin de mieux comprendre les pratiques des jeunes et de s'interroger sur la façon dont les acteurs du système éducatif les prennent en compte pour transformer, ou pas, leur métier (la troisième tâche).

Grâce à cette cartographie des usages numériques formels et informels des jeunes du collège à l'université, ainsi que des usages numériques de leurs enseignants, nous pourrons déterminer si l'École (au sens large, de la $6^{\mathrm{e}}$ à M2) s'est emparée du numérique et si les jeunes apprennent (ou, au minimum, ont la sensation d'apprendre) avec le numérique. La partie empirique de cette étude est basée sur un pluralisme méthodologique (Becker, 2013; Bernard et Joule, 2005) constitué comme nous le précisons ci-dessous.

\section{Première phase :étude qualitative pour l'élaboration d'un questionnaire optimisé (phase exploratoire)}

À partir d'un guide d'entretien à six thématiques principales (cadre familial et classe sociale, équipements et outils numériques, activités numériques personnelles, perceptions du numérique personnel, activités numériques scolaires, perceptions du numérique éducatif) portant sur environ 35 items suivant l'âge, nous avons pu analyser les pratiques numériques scolaires et extrascolaires et les perceptions de plusieurs élèves et étudiants par niveaux, de la $6^{\mathrm{e}}$ à Master 2. Sur ces 12 niveaux scolaires, 41 entretiens exploratoires semi-directifs ont ainsi été menés, ce qui représente 30 heures de transcriptions.

\section{Deuxième phase : étude quantitative}

L'analyse thématique et de sens de l'étude qualitative a permis de révéler 99 items à étudier correspondant à nos choix d'analyse. Nous avons ainsi pu supprimer des questions inutiles, reformuler des questions 
peu explicites (par exemple, le mot « visioconférence » est mal compris par les collégiens : ils utilisent par contre l'expression « faire une cam ». Il en va de même pour le verbe « submerger ", souvent incompris en $6^{\mathrm{e}}$ ) et ajouter des questions issues d'usages découverts en entretien (par exemple : le téléchargement de supports de cours numériques sur Internet « mieux faits » et plus faciles à utiliser en mobilité).

En ce qui concerne le protocole, nous souhaitions éliminer autant que possible les biais et le rendre robuste autour d'une population mère raisonnable (< 1000 étudiants à sonder). Nous avons donc mis en place un questionnaire basé sur la solution Sphinx mobile. La collecte s'effectue à l'aide d'un parc de tablettes tactiles, en mode « hors ligne » lors de l'enquête, avec synchronisation automatique à postériori. Cela nous permet de nous affranchir des lacunes ou faiblesses du WiFi (surtout en collège et lycée) ou le manque de salles informatiques, d'apporter une excellente ergonomie visuelle et fonctionnelle, d'économiser un temps précieux et, au final, d'obtenir un fort taux de remplissage du questionnaire. Les enquêtes se sont déroulées en classe complète, sur temps scolaire (durant un cours choisi avec la direction), pour éliminer le biais du « répondant volontaire » et avoir un taux de participation relevant davantage d'un recueil « exhaustif » que d'un simple échantillonnage ${ }^{3}$. La population mère a été choisie avec le $\mathrm{DASEN}^{4}$ de Pau et les chefs des établissements identifiés, de telle manière à représenter la classe sociale moyenne la plus représentative du système scolaire français, en école publique, hors zone défavorisée, hors filière spécialisée en informatique et avec un classement $\mathrm{PCS}^{5}$ moyen. Deux collèges, deux lycées et l'université de Pau ont été ainsi choisis, puis deux classes par niveaux scolaires sur les mêmes critères (50 à 70 élèves environ par niveau, sauf en licence où l'effectif est plus important) ont été sondées de manière quasi exhaustive, de la $6^{\mathrm{e}}$ à $\mathrm{M} 2$ pour un total de 792 répondants. Le tableau 1 représente la répartition des répondants par niveau.

\section{Tableau 1}

Effectifs et répondants par niveau.

\begin{tabular}{c|c|c|c}
\hline Niveau & Répondants & Effectifs & $\%$ répondants \\
\hline $6^{\text {e }}$ & 52 & 55 & $94,5 \%$ \\
\hline $5^{\mathrm{e}}$ & 48 & 57 & $84,2 \%$ \\
\hline $4^{\mathrm{e}}$ & 55 & 57 & $96,5 \%$ \\
\hline $3^{\mathrm{e}}$ & 50 & 52 & $96,2 \%$ \\
\hline $2^{\text {de }}$ & 62 & 65 & $95,4 \%$ \\
\hline $1^{\text {re }}$ & 56 & 66 & $84,8 \%$ \\
\hline Term. & 48 & 58 & $82,8 \%$ \\
\hline $\mathrm{L} 1$ & 75 & 132 & $56,8 \%$ \\
\hline $\mathrm{L} 2$ & 108 & 156 & $69,2 \%$ \\
\hline $\mathrm{L} 3$ & 110 & 129 & $85,3 \%$ \\
\hline M1 & 70 & 79 & $88,6 \%$ \\
\hline M2 & 58 & 73 & $79,5 \%$ \\
\hline Total & 792 & 979 & $80,9 \%$ \\
\hline & \multicolumn{3}{|c}{}
\end{tabular}

Enfin, une enquête en ligne, auprès des enseignants des mêmes classes (152 réponses), permet de croiser les perceptions des enseignants et de leurs apprenants. 
L'ensemble de ce travail est réalisé dans le cadre d'un sujet de thèse, que nous menons en « sociologie du numérique dans le système scolaire ", à visée plus large que le cadre de cet article. Selon notre opinion, bien que la production de ces résultats n'ait pas été prévue spécifiquement et uniquement pour répondre à la problématique de cet article, de nombreuses données prélevées sont pertinentes pour dresser un début de profil de l'apprenant avec le numérique. En conséquence, la phase qualitative exploratoire ne sera utilisée ici qu'à des fins d'illustrations et n'a été présentée que pour comprendre la genèse de la phase quantitative qui en découle.

\section{Présentation des résultats}

Nous limiterons ici la restitution de cette étude à certains résultats s'inscrivant dans la problématique de l'article, à savoir dresser un début de profil de l'apprenant numérique palois (Palois: Habitant de Pau, ville du sud-ouest de la France). Pour cela, nous apporterons à la lumière des résultats obtenus dans la phase aussi bien qualitative que quantitative, des réponses aux questions suivantes :

- Les jeunes Palois passent-ils beaucoup de temps sur écrans?

- Si oui, ont-ils la sensation d'apprendre via les écrans?

- Lorsqu'un élève apprend avec les écrans, est-ce majoritairement sous l'impulsion d'un enseignant?

- Les jeunes Palois souhaitent-ils davantage de cours sur écrans?

- Si oui, quel est le profil socioculturel de l'apprenant avec le numérique?

- L'École s'est-elle emparée du numérique pour faire apprendre?

\section{Les jeunes Palois passent-ils beaucoup de temps sur écrans?}

D'après une enquête (Common Sense Media, 2015), l'adolescent états-unien consacrerait plus d'un quart de sa journée (6 h 40) aux écrans (ordinateur, tablette, téléphone intelligent, télévision, liseuse), probablement au détriment d'autres activités de socialisation primaire. Qu'en est-il alors de l'acquisition des normes et des valeurs qui constituent le fondement de notre société, véhiculées par les parents et l'école? Cette acquisition durant la phase de socialisation primaire "méthodique » telle que décrite par Emile Durkheim dans son œuvre (1922) se ferait-elle autrement via le numérique, avec une ouverture plus précoce au monde?

Et en France? Dans notre échantillon, nous avons isolé les 13-18 ans pour assurer une juste comparaison. Nos résultats montrent un usage des écrans (tous écrans confondus) de $5 \mathrm{~h} 30$ par jour et inférieur de 70 minutes aux États-Uniens ( 6 h 40). Il est à noter que le temps moyen journalier passé sur écrans, tous âges et jours confondus est de $5 \mathrm{~h} 40$ /jour ce qui correspond peu ou prou à la norme sociale actuelle des pays développés, c'est-à-dire ayant un IDH > 0,8 (indice de développement humain). Les populations des pays dont le classement économique est plus bas sont encore plus hyperconnectées, avec 8 heures/jour en moyenne passées sur Internet, tous terminaux confondus ${ }^{6}$. 
Notre protocole nous permet de préciser ce résultat en fonction du type de jour (semaine, week-end, vacances) et du niveau scolaire.

La Figure 1 montre que la différence de temps passé sur écran est marquée entre un jour de semaine et de week-end, souvent plus de 2 heures chez les plus jeunes. Il en est de même entre un jour de weekend et un jour de vacances. La pratique numérique des collégiens et des lycéens est logiquement plus faible en semaine, puisque les écrans sont interdits en cours la plupart du temps. Cette interdiction levée, le temps passé sur écrans augmente donc rapidement dès la L1 à l'université, de 2 heures en moyenne.

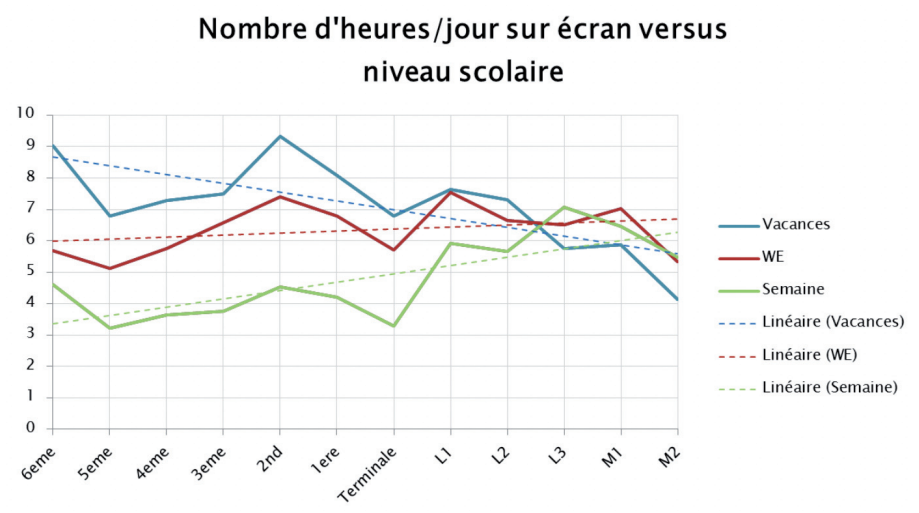

Figure 1

Nombre d'heures par jour sur écrans vs niveau scolaire.

Sur la Figure 2, la moyenne par établissement montre que le temps passé sur écrans en collège et en lycée est quasi similaire. Les différences importantes entre un jour de semaine, de week-end et de vacances disparaissent à l'université, où les écrans sont souvent autorisés en cours : la forte montée des usages numériques "plutôt » formels de semaine compense la baisse de ceux " plutôt informels » pratiqués durant les vacances. Enfin, les usages numériques « mixtes » de week-end (relevant à la fois de la sphère privée et scolaire) sont quasi identiques en volume, quels que soient le type d'établissement scolaire et l'âge. Il semblerait donc que le week-end, tous nos apprenants (élèves et étudiants) palois soient enclins à passer environ 6 h 18/jour sur écrans!

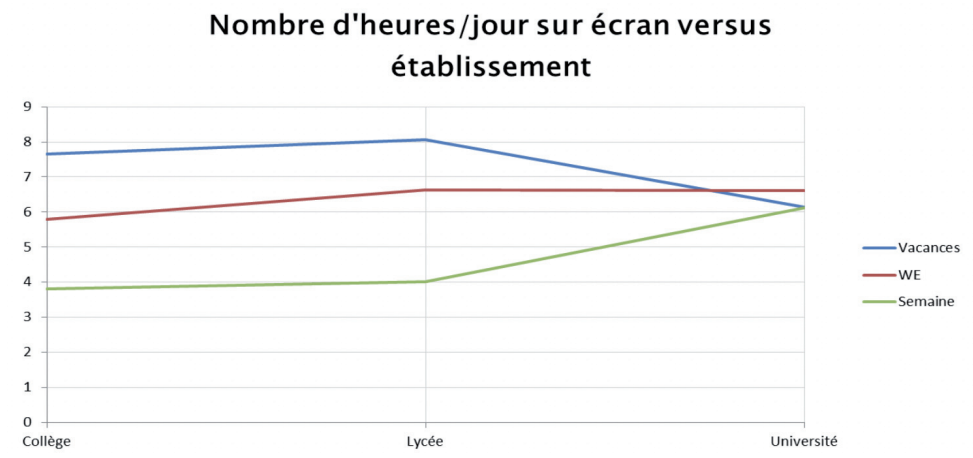

Figure 2

Nombre d'heures par jour sur écrans vs établissement. 
Prenons du recul et adoptons un point de vue annualisé. Sur les bases précédentes, la moyenne est de 2160 heures/an passées sur écrans tous niveaux confondus, ce qui correspond à 90 jours. Nos jeunes passent donc un trimestre par an sur écrans, ou autrement dit, un quart de leur vie. Une année scolaire moyenne (36 semaines de 32 heures) représente 1152 heures/an. En comparant ces chiffres de manière simpliste, nos jeunes Palois passent environ deux fois plus de temps sur écrans qu'à l'école. La place prépondérante de l'écrit et de la lecture dans notre système scolaire en font des vecteurs principaux de production des savoirs et comme nous le rappelle André Tricot en parlant du numérique et d'Internet, «le temps passé à lire par un Américain moyen a presque triplé entre 1973 et 2005, passant de $1 \mathrm{~h} 46$ en moyenne à près de $4 \mathrm{~h} 30$ de lecture quotidienne (et en excluant de ce compte toutes les activités de lecture inférieures à 1 minute) »(Tricot, 2016a, p. 48). Il est donc logique de penser que les usages numériques hors contexte institutionnel soient potentiellement sources d'apprentissages non négligeables, surtout si les usages déclarés font référence à la lecture, à la production de médias numériques ou à la consultation de sources éducatives (tutos, chaines éducatives, cours en ligne, forums thématiques...). Voyons ce qu'en pensent les principaux concernés; nous leur avons posé la question suivante : «as-tu la sensation d'apprendre beaucoup de choses à travers les écrans et Internet, que ce soit pour toi ou pour l'école?"

\section{Les jeunes Palois ont-ils la sensation d'apprendre via les écrans?}

Plus de $62 \%$ d'entre eux considèrent apprendre beaucoup ou énormément avec les écrans (respectivement 45,6 \% et 16,7 \%). Seulement 5,6 \% des répondants n'ont pas la sensation d'apprendre avec les écrans et les $28 \%$ restants ont la sensation d'apprendre un peu. Croisons ces résultats avec le niveau scolaire pour profiler notre apprenant avec le numérique. Les figures 3 et 4 (où les modalités « beaucoup » et « énormément » ont été regroupées en jaune tandis que les modalités «non » et « un peu » sont regroupées en vert) montrent que les collégiens ont peu ou pas la sensation d'apprendre via les écrans.

Les $6^{\mathrm{e}}$ et $5^{\mathrm{e}}$ sont particulièrement positionnés sur le «non ", montrant un usage plutôt ludique (jeux vidéo et usages télévisuels) pour lequel ils n’ont pas cette sensation d'apprendre souvent liée à un effort. Nous y reviendrons plus bas. Le cycle terminal universitaire, de la L3 à M2, à l'opposé, déclare une sensation très forte d'apprendre via les écrans (pour 69,2\% des étudiants). Il en est de même pour les lycéens, en tête du classement par établissement, avec une sensation forte d'apprendre avec le numérique à $77,9 \%$. 


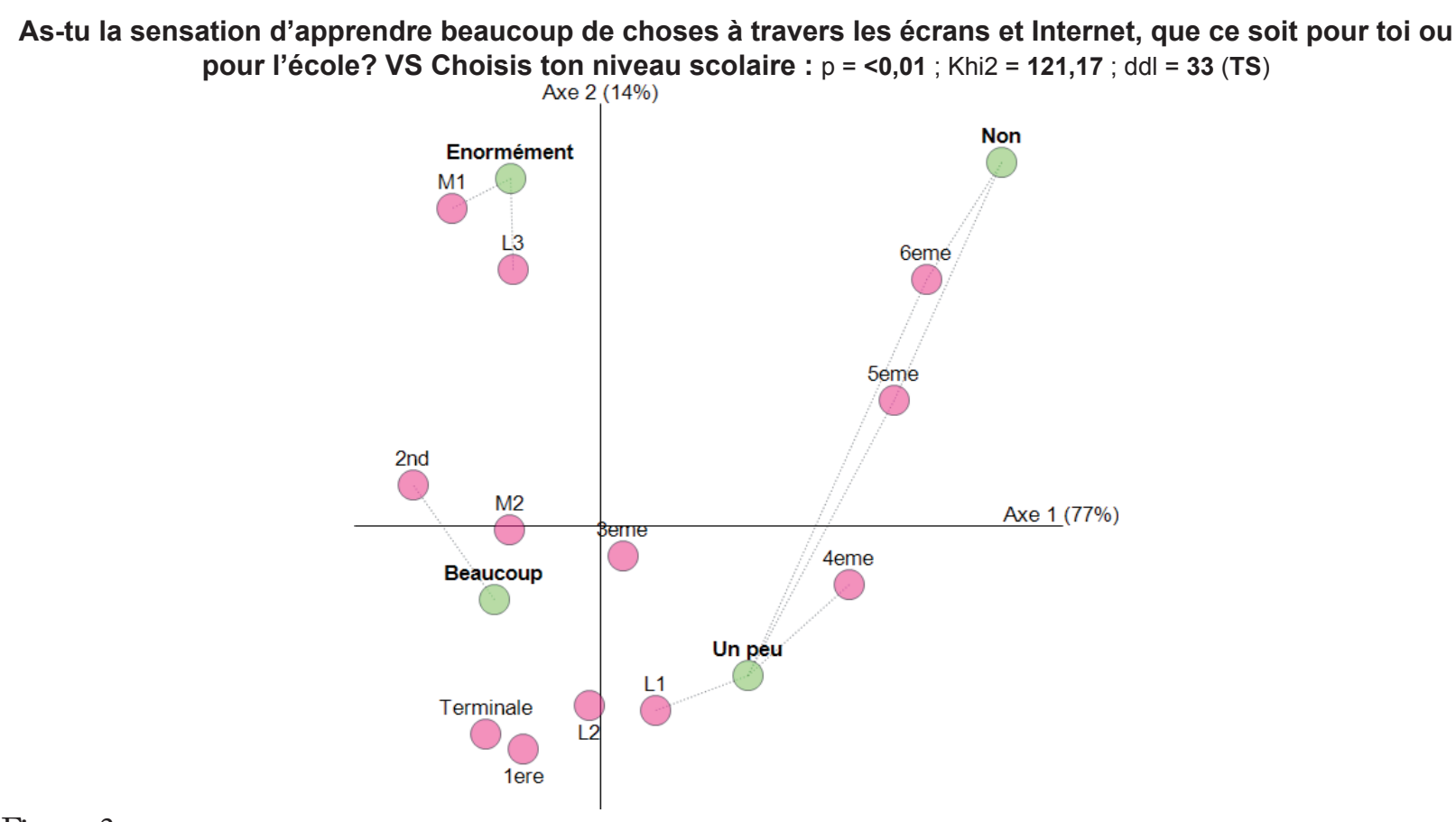

Figure 3

Sensation d'apprendre sur écrans vs niveau scolaire.

As-tu la sensation d'apprendre beaucoup de choses à travers les écrans et Internet, que ce soit pour toi ou pour l'école? VS Établissement : $p=<0,01 ; \mathrm{Khi} 2=53,64 ; \mathrm{ddl}=2$ (TS)

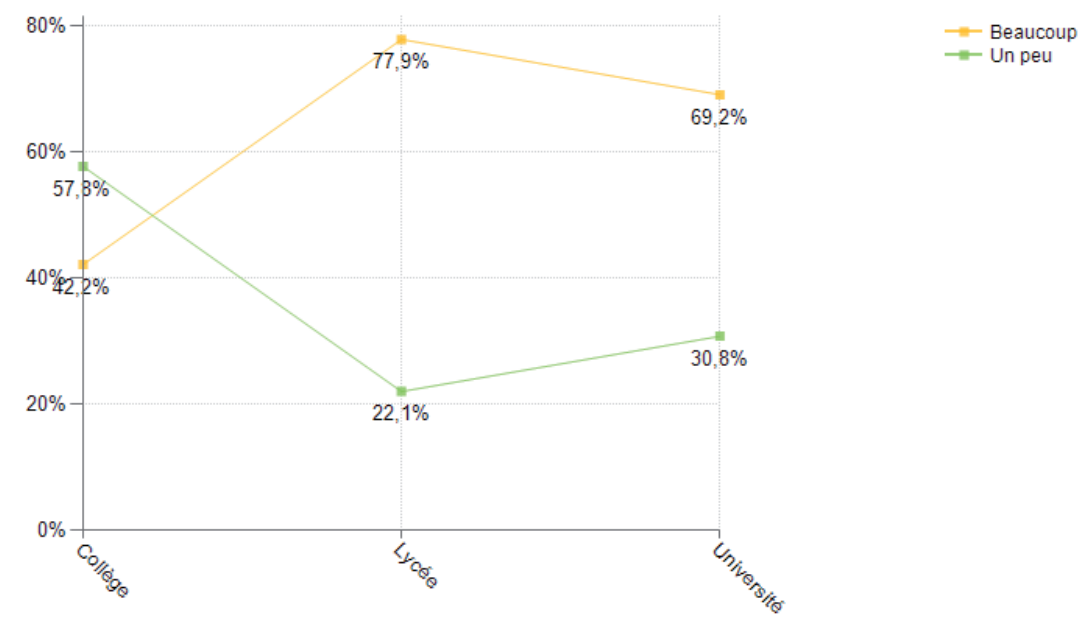

Figure 4

Sensation d'apprendre sur écrans et Internet vs type d'établissement. 
La sensation d'apprendre avec le numérique est plus faible chez les collégiens (42,2\%), et corrélée à une pratique numérique également plus faible. En effet, l'usage des écrans est bien cadré par la famille à 11 ans, beaucoup moins à 15 ans. Ce cadre familial à la baisse ainsi qu'un taux d'équipement qui progresse avec l'âge de l'élève induisent une potentielle augmentation de la pratique numérique interactive et créative et donc de la sensation d'en retirer du savoir (nous explicitons cela plus bas via le modèle ICAP [Chi et Wylie, 2014]). Nous pourrions ajouter à cela que nos jeunes élèves apprennent aussi à l'école à faire un usage pédagogique du numérique (à apprendre avec) qu'ils réinvestissent le cas échéant dans leur sphère privée (Collin, 2013). L'influence des usages du numérique est donc bidirectionnelle entre la sphère privée et scolaire.

Enfin, les usages des plus jeunes tournent le plus souvent autour de l'audiovisuel (musique, séries, dessins animés) et des jeux comme en témoigne Chloé, élève de $6^{\mathrm{e}}$ (11 ans) :

- Chloé : Bah, après moi je suis plutôt télé que l'ordinateur... donc moi, je suis souvent sur la télé. (rires)

- J.-F. : D’accord, donc si tu dois faire un choix entre la télé et l'ordinateur, tu choisis la télé?

- Chloé : Oui. (rires)

La sensation d'apprendre devant la télévision est logiquement plus faible qu'avec un écran interactif: l'apprentissage est plus manifeste lorsque l'individu est un acteur engagé dans la tâche et qu'il répond à des questions qu'il se pose plutôt que passif. Selon Dominique Bellec (2015), il existe même quatre niveaux d'engagement et d'attention (voire cinq si nous incluons le niveau «inattentif »), que nous synthétiserons ainsi :

- un premier niveau « passif» lorsque l'étudiant est attentif aux explications et accorde son attention;

- un deuxième niveau « actif » lorsqu'il réalise une activité qui lui permet de manipuler les supports d'apprentissage;

- un troisième niveau « constructif » correspondant à l'étudiant qui génère de l'information « audelà de ce qui a été enseigné »;

- un quatrième niveau « interactif », lorsque les étudiants travaillent en groupe et collaborent, en dialoguant, à une co-construction.

Selon cet ordre, chaque niveau apporte une plus-value en matière d'engagement et d'apprentissage tel que le spécifie Dominique Bellec (2015, p. 29-30) en s'appuyant sur le modèle ICAP (Chi et Wylie, 2014).

Les usages sont mécaniquement corrélés à l'équipement personnel et en ce qui concerne celui des collégiens, en $6^{\mathrm{e}}, 30 \%$ ont un ordinateur personnel contre $43,8 \%$ en $3^{\mathrm{e}}$. En ce qui concerne le téléphone portable, $40 \%$ des $6^{\mathrm{e}}$ en possèdent un, contre $89,6 \%$ en $3^{\mathrm{e}}$. Avec $81,5 \%$ d'équipement dès la $4^{\mathrm{e}}$, nous pouvons conclure que l'hyperconnexion principale via le téléphone portable est susceptible de démarrer dès l'âge de 12 ans. Les $6^{\mathrm{e}}$ sont donc peu équipés en écrans (téléphone intelligent, tablette, ordinateur) et en font un usage télévisuel plutôt passif (ce dernier résultat est issu des entretiens avec les collégiens dont un exemple est fourni plus haut), peu générateur d'apprentissages comme cela a été explicité plus haut. En $3^{\mathrm{e}}$, le cadre familial plus permissif (ou moins regardant) et l'équipement plus fréquent voient évoluer les usages vers davantage d'interactions humaines ou médiatiques, 
potentiellement génératrices d'apprentissages plus conséquents, l'interaction étant un des quatre piliers de l'apprentissage selon Stanislas Dehaene (2013). Les sensations de nos collégiens vont en ce sens. Pour finir sur cette question, nos résultats montrent que la sensation d'apprendre avec les écrans n'est pas genrée (pas de différences significatives).

\section{Lorsqu'un élève apprend avec le numérique, est-ce majoritairement sous l'impulsion d'un enseignant?}

Nous avons établi que nos jeunes Palois utilisent les écrans en moyenne 5 h 40/jour et qu'ils avaient une sensation, plus ou moins affirmée suivant le niveau scolaire, d'apprendre par leur intermédiaire. Mais quelle est la part ressentie, correspondant aux usages numériques en contexte institutionnel (le numérique éducatif)?

Nous avons posé la question suivante à nos apprenants (Figure 5) :

Quand tu apprends des choses à travers les écrans et Internet : Le plus souvent, est-ce par curiosité personnelle (ta vie privée) ou est-ce en réponse à un travail demandé par un enseignant (ta vie scolaire)?

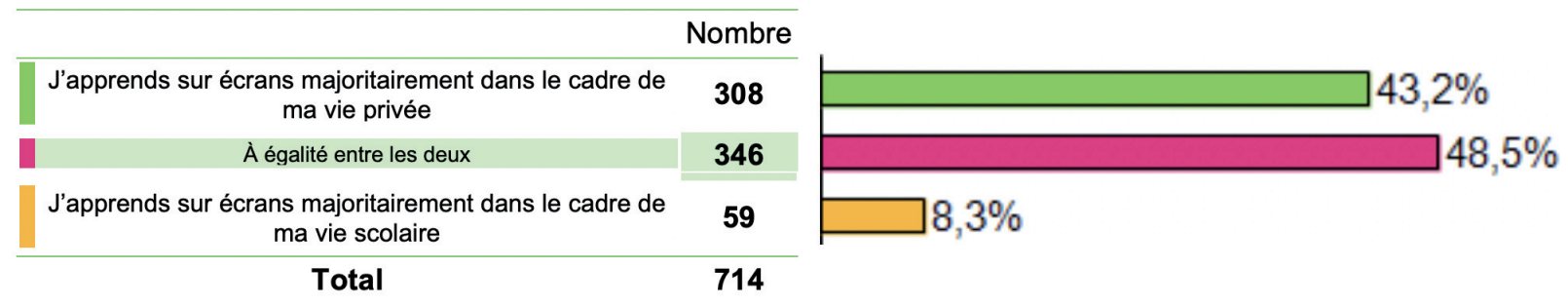

Figure 5

Sensation d'apprentissage formel ou informel.

Parmi les répondants (90\%), une petite moitié $(48,5 \%)$ ne sait pas trancher et considère que lorsquelle apprend avec le numérique, cela relève à parts égales du cadre formel et informel. L'autre moitié affiche majoritairement une sensation d'apprentissage avec le numérique plutôt informelle à 43,2\% (contre $8,3 \%$ pour le cadre formel). Cela montre que la sensation d'apprendre avec le numérique relève plutôt du contexte non institutionnel, tout comme la pratique numérique globale (OCDE, 2008). De plus, à la question «combien d'heures par semaine, toutes les activités que tes enseignants te font faire sur écrans représentent-elles environ? ", $71 \%$ des répondants déclarent moins de 4 heures/semaine. Nous avons vu que l'apprenant moyen passe $5 \mathrm{~h}$ 40/jour sur écrans. Si nous ne prenons en compte que les jours de semaine, pour nous faire une idée simple, 4 heures en moyenne sont consacrées à des activités scolaires sur écrans, contre 28 h 20 ( $5 \times 5$ h 40) pour des activités plutôt informelles sur écrans. Nous devons nuancer ces chiffres en précisant que : toutes les activités scolaires avec le numérique ne sont pas forcément porteuses d'apprentissages malgré l'intention pédagogique; toutes les activités numériques qui relèvent de la sphère privée ne découlent pas forcément d'une intention d'apprentissage. Nous comparons simplement deux temporalités « capacitantes » en matière d'apprentissages avec le numérique comme support et les sensations 
qui en découlent pour l'apprenant. Ainsi, cela nous permet d'expliquer en partie pourquoi la sensation d'apprendre avec le numérique relève plutôt de la sphère privée, car il y a fondamentalement une forte différence quantitative de pratique (un pour sept, juste en semaine et cela se creuse le week-end ou pendant les vacances)!

Voyons en quelle classe est notre apprenant avec le numérique sur la base de cette sensation d'apprentissage formel ou informel. En moyenne, la sensation d'apprentissage formel (courbe jaune) oscille entre $13,6 \%$ en collège et 6,7 \% à l'université, avec une moyenne globale à 8,3 \% (figure 6).

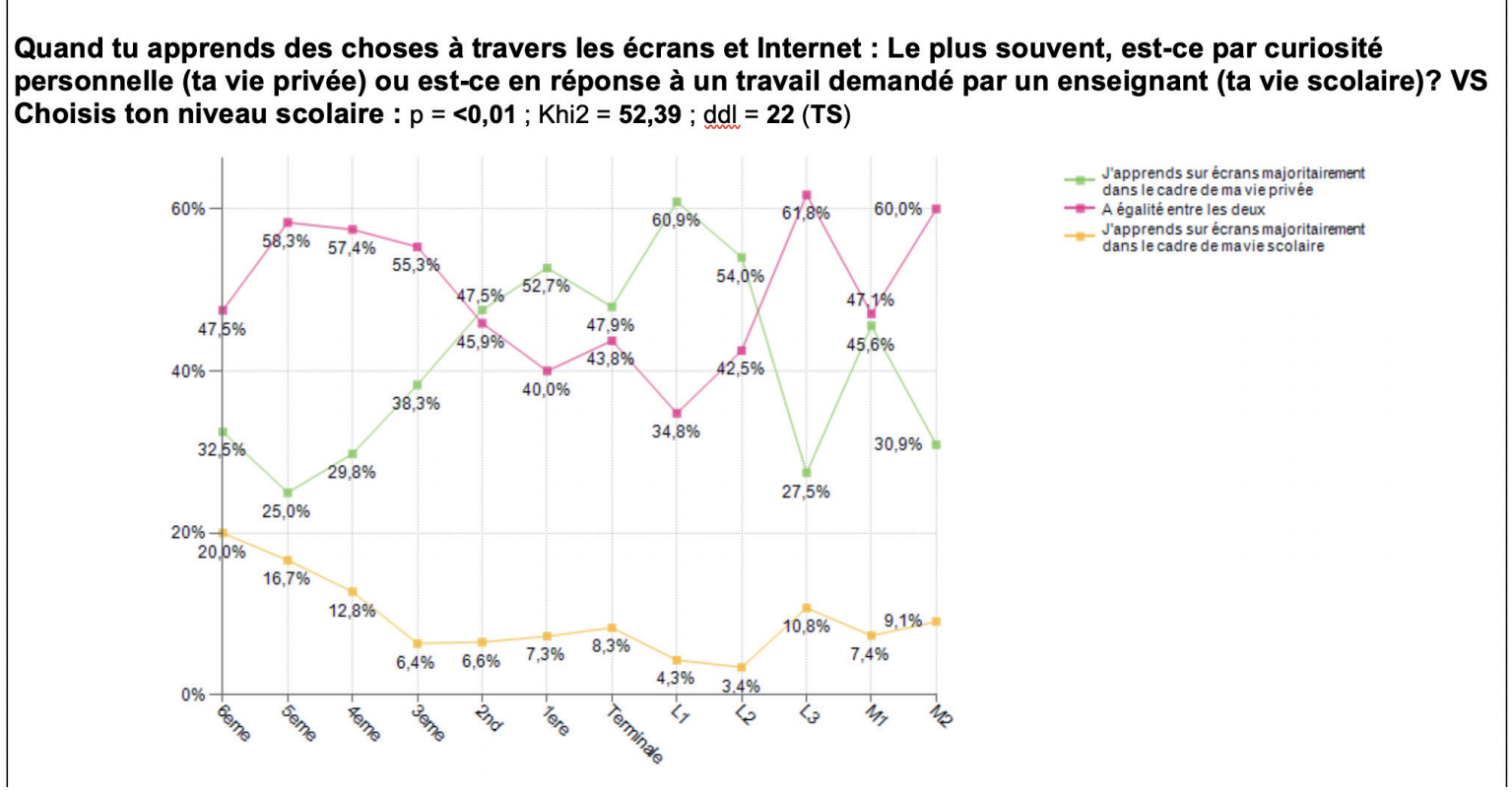

Figure 6

Sensation d'apprentissage formel ou informel vs Niveau.

À l'opposé, la sensation d'apprentissage informel (courbe verte) culmine en tête au lycée et en début d'université, en L1 et L2. Nous avons cherché à comprendre la brusque variation de cette courbe verte en L3 et en M2, pour détecter un éventuel « effet promo » et voici un début d'explication : en L3, l'échantillon est constitué de 85 étudiants en droit, 8 étudiants en géographie et 17 étudiants en histoire. La forte prédominance des étudiants en droit pourrait être à l'origine de ce pic. En effet, il ressort des entretiens que le numérique est un outil du quotidien des jeunes juristes pour accéder à de nombreuses ressources numériques indispensables : le Code civil, les textes fondamentaux, le Journal officiel, les lois et décrets, la jurisprudence, etc. Pour autant, les cours sont souvent transmissifs, ce qui ne fait pas basculer la sensation d'apprendre avec le numérique majoritairement vers le cadre formel ni même atteindre un équilibre. Enfin en M2, les enseignements devenant de plus en plus pratiques, de nombreux logiciels métiers ou bureautiques sont utilisés pour les études, la rédaction du mémoire de stage et la recherche bibliographique. Cette explication est aussi valable en L3, pour les formations exigeant un mémoire. 
Enfin, en croisant cette variable avec le genre, nous pouvons constater que 50,7 \% des garçons (versus $38,1 \%$ des filles) déclarent apprendre sur écrans majoritairement dans leur vie privée, 40,6\% des garçons (versus $54 \%$ des filles) choisissent l'égalité entre le formel et l'informel. Quant à la modalité "j'apprends sur écrans majoritairement dans le cadre de ma vie scolaire », elle n’est pas genrée avec $8 \%$ pour les filles et $8,7 \%$ pour les garçons. Donc, nous ne constatons pas de différence de genre sur la population qui pense apprendre majoritairement dans la sphère scolaire, l'apprentissage informel serait par contre plutôt masculin et l'égalité entre les deux sphères serait plutôt féminine.

Pour conclure sur cette question, l'apprentissage avec le numérique en contexte institutionnel semble peu développé ou à tout le moins peu ressenti par les apprenants, particulièrement les lycéens et en début de cycle universitaire.

\section{Les jeunes Palois souhaitent-ils davantage de cours sur écrans?}

Voyons à présent si nos jeunes palois souhaitent davantage de numérique en contexte institutionnel. En effet, les écrans pourraient très bien être perçus comme des artefacts de loisirs, de socialisation. François Dubet (2014, p. 58) parle d'intégration sociale «subjective, afin que les individus se sentent membres de cette société » et l'écosystème numérique du $21^{\mathrm{e}}$ siècle jouerait ce rôle d'intégration (en substitution à la religion, notamment) et d'information personnelle, dont l'usage ne serait pas souhaité à l'école, ce qui expliquerait le déséquilibre vu précédemment. De plus, notre système éducatif subit de nombreuses injonctions depuis les débuts de l'ère numérique et, avant elle, de l'informatique à l'école (prenons en exemple le plan Informatique pour tous, lancé en 1985 par le premier ministre Laurent Fabius) : il faut installer des ordinateurs dans la classe, il faut faire de l'informatique à l'école, il faut mettre des tableaux blancs interactifs (TBI) dans toutes les classes, il faut utiliser le numérique pour mieux former, il faut apprendre à coder à l'école, etc. Cela nécessite des investissements matériels et humains conséquents : matériels, car l'achat, l'installation et la maintenance de ces artefacts numériques coutent cher; humains, car la formation à l'usage de ces artefacts prend du temps, mais aussi des fonds publics. Ce dernier point est d'ailleurs souvent le plus négligé, ce qui représente un frein important à l'adoption du numérique éducatif par les enseignants :

Sur le terrain cependant, la pression exercée sur les milieux éducatifs pour produire rapidement des EIAH de même que le manque de formation des formateurs en technopédagogie conduisent souvent ces derniers à opter pour des solutions reflétant une pédagogie essentiellement transmissive. (Basque, Dao et Contamines, 2005, p. 178)

Ces investissements humains et financiers sont-ils souhaitables, si nos apprenants rejettent le numérique éducatif scolaire, sans parler pour l'heure de son acceptation par les enseignants? Nous ne prétendons pas répondre à cette question dans le cadre d'un tel article, mais aider à en cerner les prémices et contours en posant directement la question aux principaux concernés, les apprenants (figure 7) : 


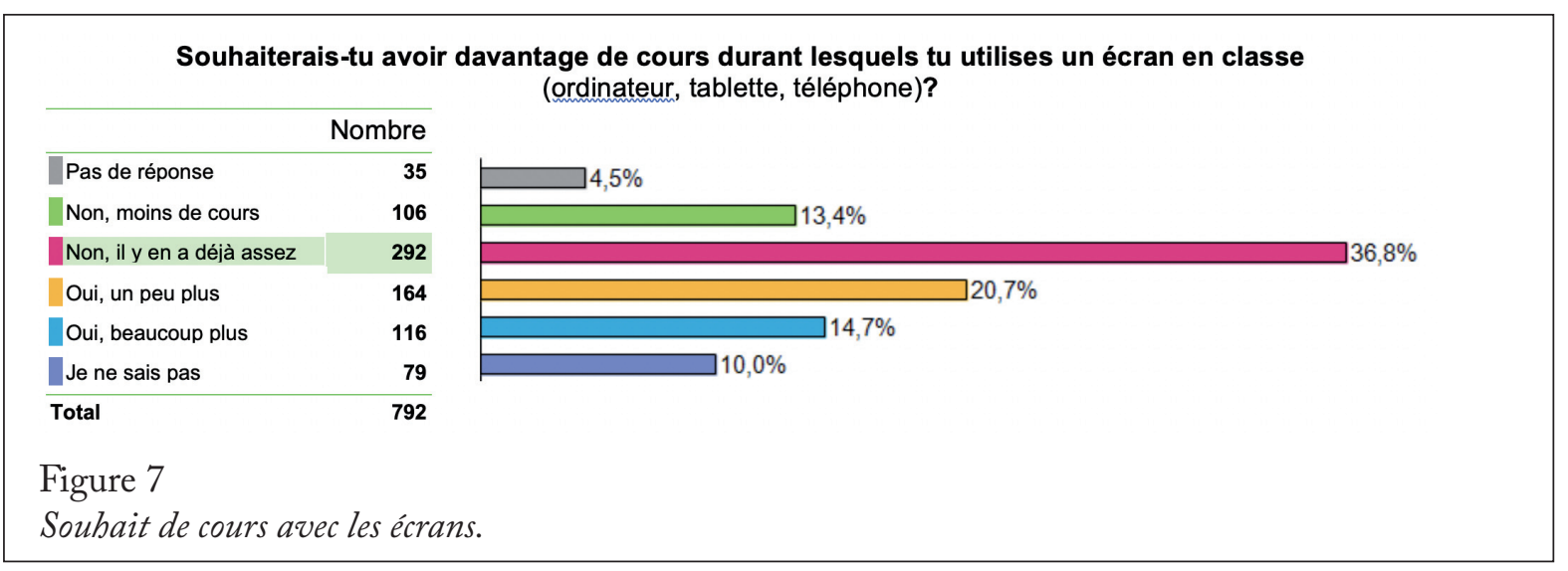

La modalité la plus représentée, à 36,8 \%, est le «non, il y en a déjà assez » qui, combinée au «non, moins de cours ", représente la moitié des répondants. Pour le reste, $14,5 \%$ ne sait pas ou ne répond pas à la question et 35,4\% des répondants souhaitent travailler davantage sur écrans en classe.

Pourrions-nous adopter une vision positive en interprétant le «non, il y en a déjà assez » comme de la satisfaction pour le système de formation en place avec le numérique? Rappelons que l'écrasante majorité de notre échantillon ressent 2 à 4 heures par semaine de pratiques numériques proposées par les enseignants. Ces pratiques se répartissent selon la Figure 8 autour de la création de textes, de la lecture de documents numériques, de vidéos, de sons et de logiciels métiers. Les autres usages relevant de l'interaction, du jeu et de la création sont très peu représentés, ce qui est d'autant plus regrettable puisqu'ils sont reconnus mobiliser au mieux les trois moteurs de l'apprentissage (l'interaction, la découverte de l'environnement et le jeu), moteurs par lesquels apprendre se fait plus facilement (Tricot, 2016b, p. 36).

\begin{tabular}{|c|c|c|}
\hline \multicolumn{3}{|c|}{$\mathrm{Nb}$} \\
\hline Non réponse & 17 & $12,1 \%$ \\
\hline Creer des documents textes & 516 & $65,1 \%$ \\
\hline |Lire des documents numériques & 497 & $62,7 \%$ \\
\hline |Regarder des vidéos & 388 & $49,0 \%$ \\
\hline Utiliser des logiciels pour leur matière (calcul, tracé de courbes, simulation...) & 385 & $\square 48,6 \%$ \\
\hline Ecouter des bandes sonores (émission radio, musique...) & 272 & $34,3 \%$ \\
\hline |Répondre à des quiz (question à choix multiple) & 198 & $25,0 \%$ \\
\hline Créer/compléter un site internet, blog & 125 & $\square 15,8 \%$ \\
\hline |Créer des documents sons/vidéos & 108 & $\square 13,7 \%$ \\
\hline Dessiner sur ordinateur & 49 & $\square 6,1 \%$ \\
\hline Dialoguer sur des réseaux sociaux & 40 & $5,0 \%$ \\
\hline |Jouer à des jeux vidéo pour apprendre & 33 & $\square 4,2 \%$ \\
\hline Dialoguer avec des correspondants d'autres classes (France ou étranger) & 26 & $3,3 \%$ \\
\hline Total & 792 & \\
\hline \multicolumn{3}{|l|}{ Figure 8} \\
\hline Activités numériques en classe. & & \\
\hline
\end{tabular}


Donc pour répondre à la question ci-dessus, les forts déséquilibres de pratiques entre les sphères scolaires et privées déjà évoquées et la pauvreté des usages numériques déclarés en termes de diversité et d'intensité montrent qu'il est difficile d'interpréter le "non, il y en a déjà assez » comme de la satisfaction pour l'offre en place. Cela relèverait plutôt d'une inadaptation des usages du numérique aux activités d'apprentissage : le potentiel éducatif du numérique semble peu compris, mal et peu investi dans les dispositifs pédagogiques, ce que nous proposons d'illustrer à travers deux témoignages issus des entretiens exploratoires semi-dirigés, en commençant par Clotilde ( $6^{\mathrm{e}}, 11$ ans) :

- J.-F. : Est-ce que tu penses que ça aide à mieux apprendre [les écrans en classe]?

- Clotilde : Enfin, quand on fait des quizz, moi je pense que oui... en tout cas, dans ma tête, c'est plus clair quand je le fais en quizz que quand je le fais en leçon. Moi je trouve que c'est plus intéressant parce que... enfin les gens copient pas déjà, j'aime pas copier. (rires) [Copier au sens écrire avec un stylo]. Et c'est souvent dans la bonne bumeur quion le fait parce quion est tous ensemble, on est réunis.

Ce témoignage militerait pour la mise en activité de l'apprenant, le travail collaboratif et l'usage de la question comme levier pédagogique (l'héritage que nous laisse Socrate va en ce sens). De plus, la recopie des cours semble mal perçue de nos jours (cela revient à plusieurs reprises dans les entretiens exploratoires, de même que la recherche sur Internet de cours équivalents au format numérique sur des sites comme Kartable ou Les bons profs, pour avoir des supports propres, plus complets et faciles à consulter dans les transports...), la transmission de supports imprimés ou dématérialisés étant si aisée et ancrée dans nos habitudes, aussi bien dans la sphère privée qu'institutionnelle. Dans le deuxième témoignage, celui de Florian ( $4^{\text {e }}, 13$ ans), nous pouvons voir que les élèves ne sont pas (tous) dupes, ni émerveillés par le numérique éducatif.

- J.-F. : Et ces activités-là, qui passent par des écrans, tu les trouves plutôt sympas, plutôt pas sympas?

- Florian : Ça dépend des activités, y en a ça va et d'autres, c'est un peu plus ennuyeux.

- J.-F. : ... Si on devait comparer les deux, est-ce que tu vas plus facilement dans un cours avec ces outils ou dans un cours classique?

- Florian : Ça dépend du cours et du prof, ça a rien à voir avec le numérique! Après, c'est mieux les profs qui utilisent le numérique.

- J.-F. : Pourquoi c'est mieux?

- Florian: Bah, l'année dernière, parce que je redouble ma quatrième, la prof de français nous montrait plein de films, c'était bien. On faisait des études de documents sur les films. Cette année, il utilise pas du tout Internet, c'est beaucoup plus ennuyeux... Il utilise que le livre.

- J.-F. : Est-ce que tu penses que, en utilisant correctement ces outils dans les cours, on peut faire des cours mieux ou au contraire ça peut déranger?

- Florian : Non, ça peut faire beaucoup mieux, ça peut intéresser beaucoup plus.

- J.-F. : Donc est-ce quion pourrait dire que tu serais plutôt pour qu'il y en ait davantage ou plutôt pour quion en mette moins?

- Florian : Non, pour qu'ils en mettent plus, mais aussi qu’ils les utilisent comme il faut...

Il nous apparait ici que les jeunes perçoivent potentiellement l'efficacité d'un cours ou d'une activité et qu'ils sont critiques quant à l'intégration du numérique en classe : oui pour le numérique éducatif, car certaines activités sont bien perçues, mais pas à tout prix. Dans la réplique «ça dépend du cours 
et du prof, ça a rien à voir avec le numérique! », nous voyons apparaitre un usage jugé peu adapté du numérique éducatif en classe. Pour autant, le numérique éducatif est un important levier motivationnel et d'engagement, comme en témoigne l'importante littérature scientifique à ce sujet, déjà abordée en introduction (Bandura et Locke, 2003; Karsenti, 2016; Romero et al., 2016). Ces témoignages pourraient donc être perçus en contradiction avec les chiffres fournis par la Figure 7, la moitié des répondants déclarant ne pas souhaiter davantage de cours intégrant une dimension numérique éducative. Alors qui sont les apprenants qui en souhaitent davantage?

\section{Quel est le profil socioculturel de l'apprenant numérique?}

En croisant divers résultats et indicateurs, nous allons tenter à présent de dégager un profil type de l'apprenant numérique, s'il existe, une personne aimant apprendre sur écrans et souhaitant voir se développer le numérique éducatif : en quelle classe est-il, quel âge a-t-il, est-ce une fille ou plutôt un garçon, est-ce un bon élève?

Commençons par le niveau scolaire grâce à la Figure 9. Pour en simplifier la lecture, nous avons regroupé les modalités « Oui, un peu plus » et "Oui, beaucoup plus » (courbe jaune « davantage »). Il apparait que les apprenants souhaitant davantage de cours instrumentés avec les écrans sont les collégiens et lycéens, sauf en terminale où une forte chute s'amorce et se confirme dans le supérieur. Notre apprenant numérique a donc plutôt moins de 17 ans (en 2017).

Souhaiterais-tu avoir davantage de cours durant lesquels tu utilises un écran en classe (ordinateur, tablette, téléphone)? VS Choisis ton niveau scolaire : $p=<0,01 ; \mathrm{Khi} 2=176,39 ; \mathrm{ddl}=11$ (TS)

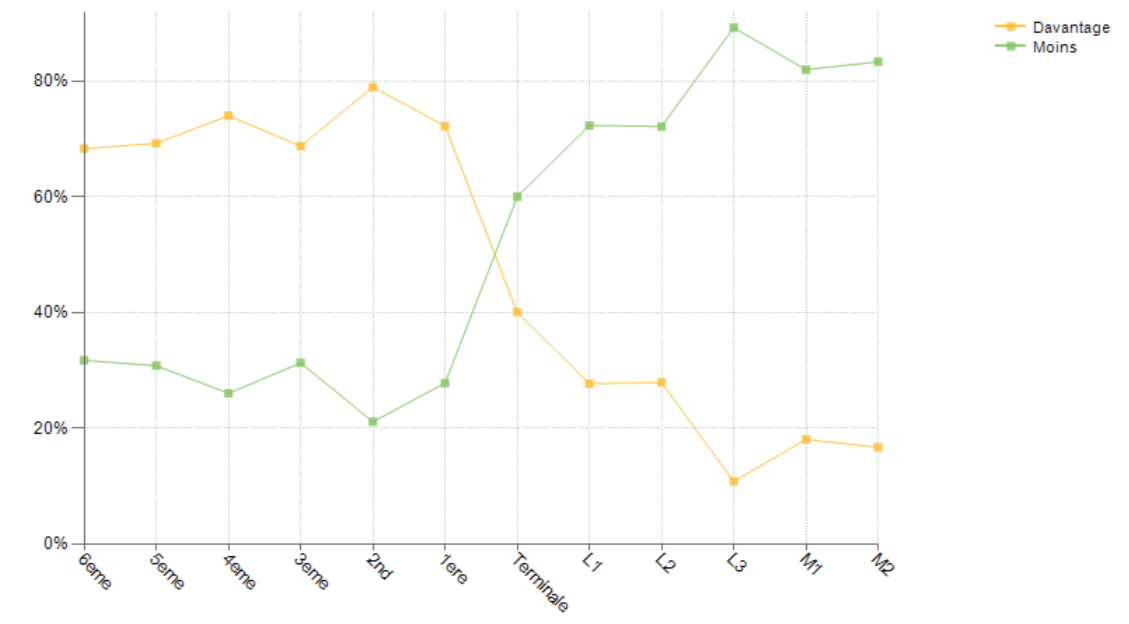

Figure 9

Soubait de cours avec les écrans vs niveau scolaire. 
Pour préciser, nous plaçons l'âge en abscisse de la Figure 10. En effet, notamment dans le supérieur, âge et niveau scolaire ne sont plus aussi intimement liés, car les redoublements se cumulent; les changements de parcours et la formation continue viennent aussi brouiller cette association. La Figure 10, bien que proche de la Figure 9, précise le pivot à 17 ans. En dessous de cet âge, le souhait de numérique éducatif en classe est très marqué, à 72,3 \% en moyenne (courbe jaune). Il chute à 43,4 \% à 17 ans puis à 19,7\% à 18 ans et plus, toujours en moyenne. D'autres données montrent que ceux qui en veulent moins sont surtout en L1 et L2 et les « satisfaits du modèle actuel » de la modalité « non, il y en a déjà assez » sont en L3, M1 et M2.

\section{Souhaiterais-tu avoir davantage de cours durant lesquels tu utilises un écran en classe (ordinateur, tablette, téléphone)? VS Quel âge as-tu? $p=<0,01 ; \mathrm{Khi} 2=171,67 ; \mathrm{ddl}=17$ (TS)}

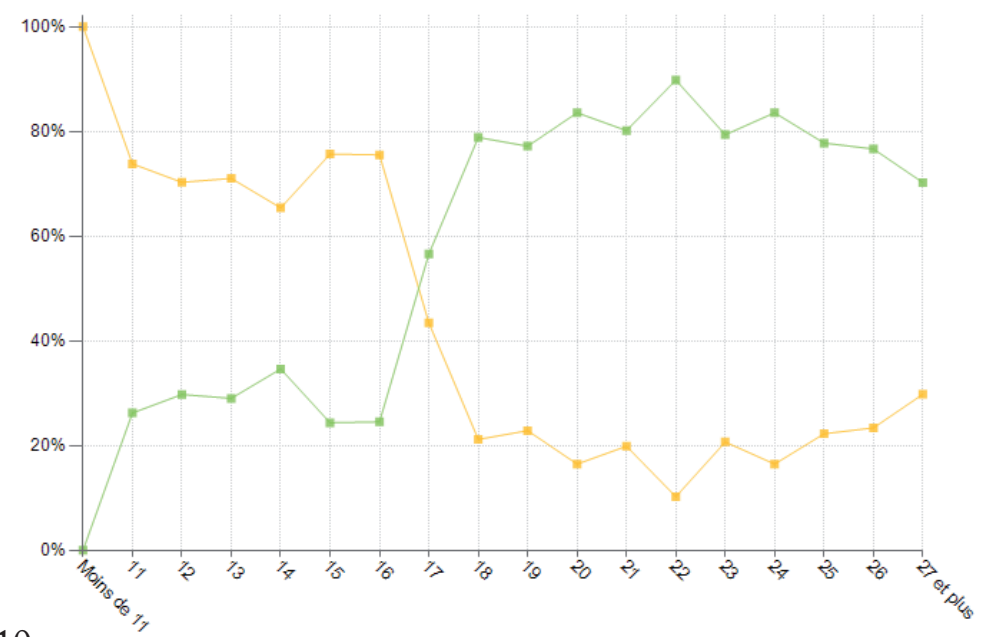

Figure 10

Soubait de cours avec les écrans vs âge.

Ce phénomène de rupture pourrait être interrogé à l'aune de deux hypothèses. La première serait que la forme scolaire, et plus précisément le passage de la "forme scolaire » à la "forme universitaire » (Cerisier, 2015; Peraya, 2018), provoquerait cette chute d'appétence pour le numérique éducatif, liée à des cours plus magistraux et à l'émancipation des étudiants en milieu universitaire. Le numérique perdrait donc de son pouvoir capacitant en amphi et de son pouvoir attracteur sur le jeune adulte dont la vie se complexifie (prédominance de la socialisation secondaire, prise de distance par rapport aux parents...) au fur et à mesure qu'il acquiert son indépendance. Cette hypothèse est sans doute active sur ce phénomène de rupture, mais l'âge moyen de passage à l'université est de 18 ans ${ }^{[7]}$, alors que notre rupture apparait à 17 ans, donc au lycée, en première et terminale. Nous y voyons donc plutôt les éléments de notre deuxième hypothèse : une interprétation " générationnelle ", c'est-à-dire une potentielle vague montante d'élèves de moins de 17 ans (en 2017), ayant une appétence forte pour le numérique éducatif. En effet, les adolescents actuels des sociétés hyperconnectées font sans doute partie de la première génération de l'humanité à s'être construits dans cette hyperconnexion (disponibilité permanente du mobile dans la poche et connectivité quasi illimitée en simultané). À la fois leur socialisation primaire (familiale) et le début de leur socialisation secondaire (école; groupe 
de pairs; sphère professionnelle, institutionnelle, associative, etc.) sont modifiées par le numérique, comme nous le laisse d'ailleurs à penser cette citation de Béatrice Mabillon Bonfils :

La socialisation des années 50 ou 60 était marquée par la structure de classes de la société française. La socialisation primaire des jeunes était donc plutôt cohérente et univoque : familles ouvrières et familles bourgeoises transmettaient des cultures de classe distinctes et segmentées [...]. La diversification des relais de socialisation primaires de plus en plus prégnants va changer la donne : notamment l'École de masse, la révolution des média, mais aussi les loisirs, les groupes de pairs. La socialisation secondaire elle-même s'est complexifiée avec la fragilisation de l'insertion professionnelle et l'éclatement de l'institution familiale. La socialisation verticale, générationnelle et « méthodique » de l'individu moderne laisse place à l'hétérogénéité croissante des cadres socialisateurs, au polythéisme de valeurs et la pluri-socialisation du sujet trans-moderne. (Bonfils, 2018, paragr. 22)

Cette situation est inédite et pourrait expliquer cette appétence pour le numérique éducatif, car nos jeunes lycéens pourraient avoir compris le potentiel éducatif de l'outil et écosystème numérique (Merzeau, 2017), se formant au quotidien de manière informelle par lui, grâce à lui et en lui. Cette hypothèse devra être vérifiée empiriquement : si lors d'une prochaine enquête en 2019, la vague se déplace à 19 ans par exemple, cela nous incitera à penser qu'une vague ou génération montante d'élèves hyperconnectés possède une appétence marquée pour le numérique en éducation. Cette vague pourrait alors déferler jusqu'en M2 en 2024 pour « envahir » l'intégralité du système scolaire.

L'appétence pour le numérique éducatif est-elle genrée? La Figure 11 montre que les filles sont presque à l'identique des garçons sur les modalités « Non, moins de cours [avec les écrans] », montrant que l'insatisfaction n'est pas genrée ici, et sur la modalité « Oui, un peu plus ». Par contre, elles sont moitié moins nombreuses à en vouloir « beaucoup plus » $(12,3 \%$ contre $24,2 \%)$, ce qui se reporte en faveur $\mathrm{du}$ « Non, il y en a déjà assez ».

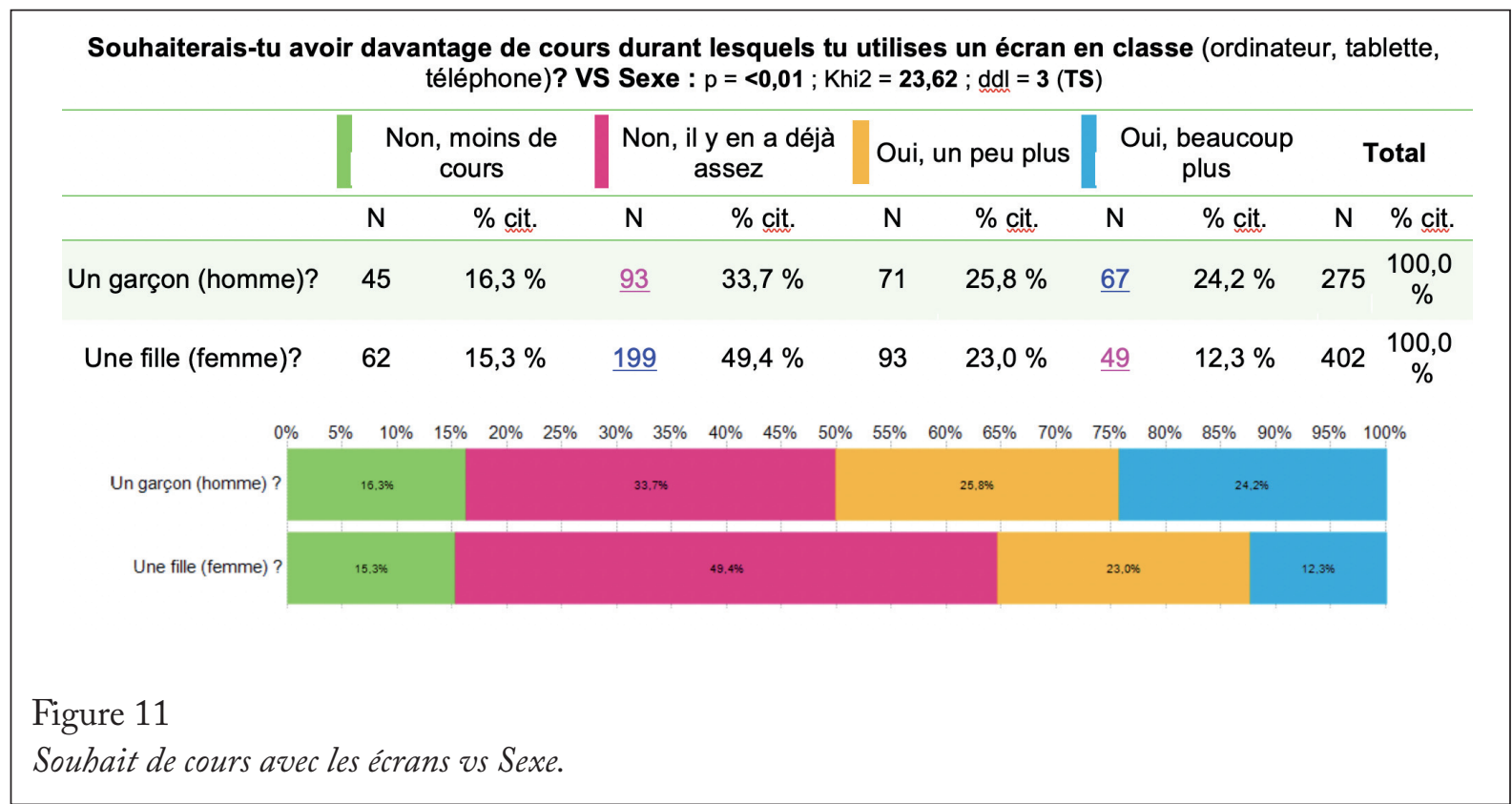


Les filles semblent donc davantage opposées à une augmentation du numérique éducatif en classe $(64,7 \%$ contre $50 \%$ pour les garçons) et satisfaites du modèle actuel, alors que pour la pratique numérique globale, nous n'avons pas relevé de différence quantitative significative : les filles passent sur écrans $7 \mathrm{~min} /$ jour en moins que les garçons, autour des $5 \mathrm{~h} \mathrm{40/jour} \mathrm{dans} \mathrm{la} \mathrm{sphère} \mathrm{privée} \mathrm{et} \mathrm{la}$ perception du nombre d'heures par semaines passées sur écrans en classe (question « combien d'heures par semaine toutes les activités que tes enseignants te font faire sur écrans représentent-elles environ? ») est très similaire entre les filles et les garçons. Notre "apprenant numérique » serait donc un peu plus fréquemment un garçon, au moins dans le contexte formel. La prédominance du genre masculin dans les activités en rapport avec la technologie est démontrée par de nombreuses recherches, y compris dans le domaine du numérique. En effet, après avoir dressé un panorama des recherches sur le genre et les TIC, Josiane Jouët conclut :

Aux hommes reviendraient des usages plus technicisés et une emprise sur les machines conforme au modèle culturel d'une conception masculine des TIC, aux femmes des usages relationnels et une mise à distance de la matérialité de ces outils. Les TIC apparaissent donc comme des opérateurs sociaux où se reconstruit le clivage du genre à l'œuvre dans la société. (Jouët, 2003, p. 81)

Cette revue de littérature sur le genre expliquerait, au moins en partie, l'appétence du genre masculin pour le numérique éducatif et les écrans, via un schéma préétabli socialement et culturellement. La sociologue conclut que « tout comme le genre, les technologies de communication sont des constructions sociales dynamiques qui ne sont pas stabilisées » (Jouët, 2003, p. 82).

Nos apprenants sont le plus souvent caractérisés par leur performance scolaire, évaluée par des notes. Nous avons demandé aux répondants leur moyenne générale de l'année précédente et nous allons croiser ici cet indicateur avec le souhait de numérique éducatif en classe.

Souhaiterais-tu avoir davantage de cours durant lesquels tu utilises un écran en classe (ordinateur, tablette, téléphone)? VS L'année dernière, ta moyenne générale était plutôt : $p=<0,01 ;$ Khi2 $=20,46 ; d d l$ $=4$ (TS)

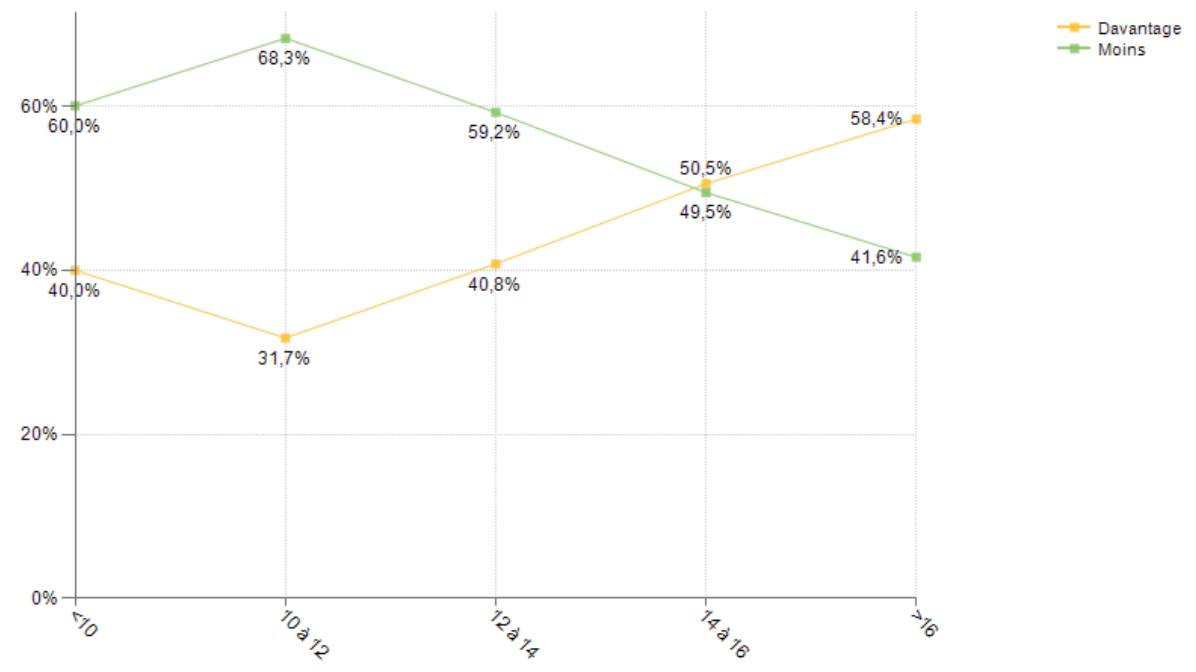

Figure 12

Soubait de cours avec les écrans vs moyenne générale. 
Il apparait que les élèves moyens (10/20 à 12/20) ont le moins d'appétence pour le numérique en classe, alors que les meilleurs sont les plus demandeurs et se positionnent majoritairement pour davantage de cours avec les écrans à partir de 14/20 de moyenne générale. Le numérique éducatif serait-il souhaité surtout par les bons apprenants? Cette hypothèse est convergente avec d'autres, comme le mentionne une méta-analyse portant sur l'efficacité des TIC en éducation, en algèbre ici : « les élèves qui travaillent dans un environnement informatisé atteignent un meilleur niveau de raisonnement que les autres, mais les TIC semblent être d'autant plus bénéfiques que les élèves sont considérés comme bons » (Thibert, 2012, p. 5). Le même article montre qu'il existe aussi de très nombreuses recherches montrant l'inefficacité des TIC sur l'apprentissage, ou du moins qui n'arrivent pas à en démontrer l'efficacité. La prudence est de mise. Telle est la conclusion du dernier livre d'André Tricot, à propos des innovations pédagogiques avec les outils numériques : «il est tout à fait impossible de savoir aujourd'hui si elles améliorent les apprentissages des élèves de façon générale » (Tricot, 2017, p. 150)!

\section{Conclusion}

Cette étude confirme que les jeunes Palois sont hyperconnectés avec une moyenne de $5 \mathrm{~h}$ 40/jour consacrées aux écrans, ce qui correspond bien à la norme sociale actuelle des pays comme la France ${ }^{[6]}$. En tenant compte du nombre croissant des activités numérisées et d'une technologie de plus en plus accessible, nous aurions tendance à penser que cela pourrait s'accroitre, sur le modèle des adolescents états-uniens (6 h 40/jour).

Les jeunes Palois considèrent les écrans comme une source d'apprentissages très importante, surtout au lycée et de la L3 à M2, sans différence de genre. L'équipement n'est pas discriminant sur ces aspects ou à la marge.

De manière globale, la sensation d'apprendre avec le numérique relève très majoritairement de la sphère privée et de l'apprentissage informel, tout comme la pratique numérique globale. Cela confirme ce que nous évoquions en introduction et le numérique n'en est qu'un prolongement : la plus grande partie des connaissances et des savoir-faire qu'acquiert un individu au cours de son existence se fait au moyen de ce mode d'éducation informelle (Hamadache, 1993). L'apprentissage avec le numérique en contexte institutionnel semble donc peu développé ou à tout le moins peu ressenti par les apprenants, particulièrement les lycéens et en début de cycle universitaire.

Les répondants déclarent pour moitié ne pas souhaiter davantage de cours intégrant une dimension numérique éducative. Pourtant, le numérique éducatif est reconnu comme un important levier motivationnel et d'engagement, comme en témoigne l'importante littérature scientifique à ce sujet, ce qui peut sembler paradoxal. Alors qui sont les apprenants ayant de l'appétence pour le numérique éducatif? Nous avons donc tenté de dresser un début de profil de l'apprenant avec le numérique, c'est-à-dire l'élève ou l'étudiant ayant une forte sensation d'apprendre via les écrans, ainsi qu'une forte appétence pour le numérique en classe.

Ce profil, que nous avons nommé par commodité « apprenant numérique », désigne les élèves qui seront manifestement demandeurs et consommateurs d'une pédagogie appuyée sur le numérique. Selon les résultats discutés plus haut, notre "apprenant numérique » serait donc plutôt un garçon, au moins dans le contexte formel, avec de bons résultats scolaires (14/20 et plus). Il apprend beaucoup sur écrans 
et est au lycée ou en cycle terminal universitaire (L3, M2). Comme il souhaite voir le numérique se développer en classe, il a moins de 17 ans. Le croisement de ces deux conditions isole donc la population des $2^{\text {de }}$ et $1^{\text {re }}$ de lycée, principal « vivier » de notre apprenant numérique.

Nous pensons donc que nos jeunes lycéens pourraient avoir compris le potentiel éducatif de l'outil et écosystème numérique (d'où leur appétence), se formant au quotidien de manière informelle par lui, grâce à lui et en lui. Ils pourraient également faire partie d'une vague montante d'élèves à la fois byperconnectés, ayant de l'appétence pour le numérique en classe et appartenant au profil "apprenants numériques ", que l'École aurait tout intérêt à former avec davantage de pratiques numériques formelles (ils entreront à l'université en 2019). Cette vague pourrait alors déferler jusqu'en M2 en 2024 pour «envahir " l'intégralité du système scolaire. Cette hypothèse devra être vérifiée empiriquement lors d'une prochaine enquête en 2019 pour valider le déplacement de cette vague. Nous pourrions nuancer ces propos en arguant d'une carence de pratiques numériques formelles en collège et lycée, conduisant à un désir plus fort par simple phénomène de manque. Ces résultats préliminaires et hypothèses ouvrent donc la voie vers d'autres études en ce sens.

Alors à l'aune de ces quelques résultats, le système éducatif s'est-il emparé du numérique pour faire apprendre? $\mathrm{Vu}$ la faiblesse ressentie des situations pédagogiques incluant le numérique (en termes de quantité et de diversité d'usages) et d'un apprentissage avec le numérique perçu comme informel, il semblerait que non. Quant à la "mécanisation de l'éducation" évoquée en introduction, la « mécanisation par le numérique », bien que souvent redoutée, ne semble ni effective ni souhaitée, sauf peut-être chez nos apprenants numériques.

\section{Notes}

1 Pour fluidifier la lecture, nous ne ferons pas de distinction de genre. Par exemple, le mot « collégiens » renverra aux élèves du collège, qu'ils soient féminins ou masculins.

2 Pour simplifier le discours, nous ne distinguerons pas les apprentissages non formels (clubs, pratiques associatives régulières...) des apprentissages informels selon la classification proposée par Ali Hamadache (1993) dans le rapport de I'UNESCO.

3 Nos 792 répondants représentent, en effet, 80,9\% de l'effectif total des classes choisies, avec un taux de participation de 92,8 \% en collège, de 86,8 \% en lycée et de $74 \%$ à l'université (ou l'absentéisme est plus fort!).

4 DASEN : directeur académique des services de l'Éducation nationale. Il est chargé d'animer et de mettre en œuvre la politique éducative dans son département.

5 PCS : nomenclature des professions et catégories socioprofessionnelles, remplaçant le CSP.

6 Source We Are Social: https://wearesocial.com/fr/blog/2017/01/digital-social-mobile-les-chiffres-2017

7 Source ministérielle 2016 : http://cache.media.education.gouv.fr/file/2017/90/6/depp-rers-2017-donnees-fiche-0811_810906.xls

\section{Références}

Bandura, A. et Locke, E. A. (2003). Negative self-efficacy and goal effects revisited. Journal of Applied Psychology, 88(1), 8799. http://dx.doi.org/10.1037/0021-9010.88.1.87 
Basque, J., Dao, K. et Contamines, J. (2005, mai). L'apprentissage « situé» dans les cours en ligne : le cas du colloque scientifique virtuel (CSV). Communication présentée à la conférence EIAH 2005 (Environnements informatiques pour l'apprentissage humain), Montpellier. Repéré à https://telearn.archives-ouvertes.fr/hal-00005660/document

Becker, H. S. (2013). Les ficelles du métier : comment conduire sa recherche en sciences sociales. Paris : La Découverte.

Bellec, D. (2015). Apprentissages par enseignement à partir d'environnements complexes : effets de l'isolement des éléments en interaction et du séquencement de la présentation (Thèse de doctorat, Université Toulouse le Mirail - Toulouse II). Repéré à https://tel.archives-ouvertes.fr/tel-01284008/document

Bernard, F. et Joule, R.-V. (2005). Le pluralisme méthodologique en sciences de l'information et de la communication à l'épreuve de la « communication engageante ». Questions de communication, (7), 185-208. http://dx.doi.org/10.4000/questionsdecommunication.4647

Bonfils, B. M. (2018). L'école est finie! Lère trans-moderne du savoir-relation et la fin de la transmission? Éducation et socialisation. Les cabiers du CERFEE, (47). http://dx.doi.org/10.4000/edso.2862

Buisson, F. (dir.) (1911). Nouveau dictionnaire de pédagogie et d'instruction primaire. Repéré à http://www.inrp.fr/edition-electronique/lodel/dictionnaire-ferdinand-buisson/

Cerisier, J.-F. (2015). La forme scolaire à l'épreuve du numérique. http://dx.doi.org/10.13140/RG.2.1.2208.5848

Chi, M. T. H. et Wylie, R. (2014). The ICAP framework: Linking cognitive engagement to active learning outcomes. Educational Psychologist, 49(4), 219-243. http://dx.doi.org/10.1080/00461520.2014.965823

Collin, S. (2013). Saisir les usages numériques éducatifs des élèves dans leur globalité. Formation et profession, 21(2), 105108. http://dx.doi.org/10.18162/fp.2013.a23

Collin, S., Guichon, N. et Ntebutse, J. G. (2015). Une approche sociocritique des usages numériques en éducation. STICEF, (22), 89-117. http://dx.doi.org/10.3406/stice.2015.1688

Common Sense Media. (2015). The Common Sense census: Media use by tweens and teens, 2015. Repéré à https://www.commonsensemedia.org/research/the-common-sense-census-media-use-by-tweens-and-teens

Crozier, M. et Friedberg, E. (2014). L'acteur et le système, les contraintes de l'action collective. Paris : Seuil.

De Munck, J. (2011). Les trois dimensions de la sociologie critique. SociologieS. Repéré à https://sociologies.revues.org/3576

Dehaene, S. (2013, novembre). Les quatre piliers de l'apprentissage, ou ce que nous disent les neurosciences. ParisTech Review. Repéré à http://parisinnovationreview.com/article/les-quatre-piliers-de-lapprentissage-stanislas-dehaene

Dubet, F. (2014). La préférence pour l'inégalité : comprendre la crise des solidarités. Paris : Seuil.

Durkheim, E. (1922). Éducation et sociologie (3e éd.). Paris : Presses universitaires de France.

Hamadache, A. (1993). Articulation de l'éducation formelle et non formelle : implications pour la formation des enseignants. Repéré à http://unesdoc.unesco.org/images/0010/001001/100125f.pdf

Jouët, J. (2003). Technologies de communication et genre : des relations en construction. Réseaux, (120), 53-86. http://dx.doi.org/10.3917/res.120.0053

Karsenti, T. (2016, 18 octobre). Les technologies ont-elles un réel impact sur la réussite scolaire? [Billet de blogue]. Repéré à http://www.cforp.ca/educo/les-technologies-ont-elles-un-reel-impact-sur-la-reussite-scolaire

Latour, B. et Biezunski, M. (2010). La science en action : introduction à la sociologie des sciences. Paris : La Découverte.

Meirieu, P. (1999). Des enfants et des hommes. Littérature et pédagogie, Tome 1, La promesse de grandir. Paris : ESF.

Merzeau, L. (2017). De la bibliothèque à l'Internet : la matrice réticulaire. Dans T. Boccon-Gibod, C. Ion et É. Mougenot (dir.), Robert Damien, du lecteur à l'électeur. Bibliothèque, démocratie et autorité (p. 44-53). Villeurbanne : Presses de l'ENSSIB. 
Organisation de coopération et de développement économiques (OCDE). (2008). New millennium learners : Initial findings on the effects of digital technologies on school-age learners. Repéré à http://www.oecd.org/site/educeri21st/40554230.pdf

Peraya, D. (2018). Technologies, innovation et niveaux de changement : les technologies peuvent-elles modifier la forme universitaire? Distances et médiations des savoirs, (21). Repéré à http://journals.openedition.org/dms/2111

Romero, M., Laferriere, T. et Power, T. M. (2016). The move is on! From the passive multimedia learner to the engaged cocreator. eLearn, 2016(3). http://dx.doi.org/10.1145/2904374.2893358

Thibert, R. (2012). « Pédagogie + Numérique = Apprentissages 2.0 ». Dossier d'actualité Veille et Analyses, (79). Repéré à http://veille-et-analyses.ens-lyon.fr/DA-Veille/79-novembre-2012.pdf

Tricot, A. (2016a). Le numérique à l'école : je t'aime, moi non plus. Direction, (42), 46-48. Repéré à http://www.snpden. net/direction/adherents/pdf242/d242-metier-Observatoire-national-securite-TICE.pdf

Tricot, A. (2016b). Apprentissages scolaires et non scolaires avec le numérique. Administration É Éducation (152), 33-39. http://dx.doi.org/10.3917/admed.152.0033

Tricot, A. (2017). L'innovation pédagogique. Mythes et réalités. Paris : Retz.

\section{Pour citer cet article}

Ceci, J-F. (2019). Le profil de l'apprenant numérique, du collègue à l'université : le cas de Pau. Formation et profession, 27(3), 91-112. http://dx.doi.org/10.18162/fp.2019.485 